03

\title{
Генерация квантово-каскадного лазера с тонкой верхней обкладкой
}

\author{
(C) А.В. Бабичев ${ }^{1,2,3}$, А.Г. Гладышев ${ }^{2}$, Д.В. Денисов ${ }^{4}$, Л.Я. Карачинский ${ }^{1,2,3}$, И.И. Новиков ${ }^{1,2,3}$, \\ L. Boulley ${ }^{5}$, A. Bousseksou ${ }^{5}$, Н.A. Пихтин ${ }^{3}$, А.Ю. Егоров ${ }^{1}$ \\ ${ }^{1}$ Университет ИТМО, \\ 197101 Санкт-Петербург, Россия \\ ${ }^{2}$ ООО „Коннектор Оптикс“, \\ 194292 Санкт-Петербург, Россия \\ ${ }^{3}$ Физико-технический институт им. А.Ф. Иоффре РАН, \\ 194021 Санкт-Петербург, Россия \\ ${ }^{4}$ Санкт-Петербургский государственный электротехнический университет „ЛЭТИ“, \\ 197022 Санкт-Петербург, Россия \\ ${ }^{5}$ Center of Nanoscience and Nanotechnology (C2N), Univ. Paris Sud and Paris-Saclay, \\ 91405 Orsay cedex, France \\ e-mail: a.babichev@mail.ioffe.ru
}

Поступила в редакцию 27.02.2019 г.

В окончательной редакции 27.02.2019 г.

Принята к публикации 15.03.2019 г.

\begin{abstract}
Представлены результаты экспериментов по созданию и изучению свойств инжекционных квантовокаскадных лазеров спектрального диапазона 7-8 $\mathrm{m}$ в геометрии волновода с тонкой верхней обкладкой на основе фосфида индия. Гетероструктура сформирована методом молекулярно-пучковой эпитаксии на подложке InP с активной областью на основе гетеропары твердых растворов $\operatorname{In}_{0.53} \mathrm{Ga}_{0.47} \mathrm{As} / \mathrm{Al}_{0.48} \mathrm{In}_{0.52} \mathrm{As}$. Достигнута лазерная генерация на длине волны излучения $7.8 \mu \mathrm{m}$ при температуре $300 \mathrm{~K}$ с пороговой плотностью тока $\sim 6 \mathrm{kA} / \mathrm{cm}^{2}$. Значения характеристических температур $T_{0}$ и $T_{1}$ для исследованных квантовокаскадных лазеров составляют порядка $150 \mathrm{~K}$ и $450 \mathrm{~K}$ соответственно. Полученные результаты подтверждают перспективность конструкции волновода с тонкой верхней обкладки для устройств детектирования жидкостей, создания устройств микрофлюидики, а также фотонных схем на кремнии.
\end{abstract}

Ключевые слова: квантово-каскадный лазер, фосфид индия, МПЭ, генерация.

DOI: $10.21883 /$ OS.2019.08.48041.82-19

\section{Введение}

Инжекционные квантово-каскадные лазеры (ККЛ), излучающие в спектральном диапазоне $7-8 \mu \mathrm{m}$, могут эффективно применяться для проведения дистанционного газоанализа, в частности лазеры с длиной волны 7.3-7.9 $\mu$ т используются для детектирования $\mathrm{SO}_{x}, \mathrm{CH}_{4}$, $\mathrm{H}_{2} \mathrm{~S}, \mathrm{C}_{2} \mathrm{H}_{2}, \mathrm{~N}_{2} \mathrm{O}$, тринитротолуола [1-3]. Детектирование метана (на длине волны излучения $7.8 \mu \mathrm{m}$ ) на основе ККЛ применяется для удаленного контроля утечек на газопроводах и химических производствах.

Стандартный подход при формировании волновода ККЛ для спектроскопии и дистанционного газоанализа - увеличение толщины верхней обкладкой волновода до 3-5 $\mathrm{m}$, что позволяет уменьшить перекрытие световой волны со слоями верхней металлизации и, как следствие, уменьшить величину плотности порогового тока за счет меньших потерь в слоях металлизации. Более того, использование слоев InP взамен InAlAs при формировании верхней обкладки позволяет более эффективно отводить тепло от активной области при монтаже эпитаксиальной поверхностью на теплоотвод, что является важным для реализации лазерной генерации в непрерывном режиме. Типичная ширина спектральной линии ККЛ на полувысоте (FWHM) при работе в непре- рывном режиме накачки $<0.001 \mathrm{~cm}^{-1}$, а в импульсном режиме $\sim 0.01 \mathrm{~cm}^{-1}[4]$, что предопределяет выбор непрерывного режима при формировании источников лазерного излучения среднего ИК диапазона, требуемых для спектроскопии и газоанализа [5].

В свою очередь, существует ряд областей, где требуется применение конструкции с малой толщиной слоев верхней обкладки: детектирование осаждаемых на поверхности ККЛ жидкостей [6-8], а также формирование сенсоров для газоанализа, расположенных непосредственно на чипе. При детектировании жидкостей на поверхности ККЛ [6-8] уменьшение толщины верхней обкладки приводит к наличию „хвоста“ интенсивности излучения основной моды на поверхности гетероструктуры. В результате наблюдается изменение мнимой части показателя преломления, а не его действительной части, как в случае конструкции волновода с толстой обкладкой. Поглощение осаждаемой на поверхности ККЛ жидкости является причиной изменения величины потерь волновода ККЛ и, как следствие, длины волны лазерной генерации [6]. Использование геометрии волновода с тонкой верхней обкладкой также позволяет увеличить эффективность связывания оптической моды с волноводом, что требуется при формировании сенсоров 
для газоанализа, расположенных непосредственно на чипе. Первые результаты по формированию фотонных схем на основе каскадных лазеров при расположении на кремнии, а также по интеграции ККЛ с InGaAsволноводами представлены в работах [7-10] и $[11,12]$ соответственно. Ранее показано, использование конструкции волновода без верхней обкладки при формировании сплошной верхней металлизации не позволяет реализовать комнатную генерацию. Предполагается, что использования конструкции с тонкой верхней обкладкой позволят уменьшить потери, связанные с расположением металла над активной областью, что позволит реализовать работу при комнатной температуре.

В настоящей работе представлены результаты по формированию и исследованию оптических характеристик инжекционных ККЛ с тонкой верхней обкладкой на основе InP.

\section{Эксперимент}

Гетероструктура ККЛ выращена компанией „Коннектор Оптикс“ с использованием промышленной установки молекулярно-пучковой эпитаксии (МПЭ) Riber 49, оснащенной твердотельным источником мышьяка крекерного типа и источниками марки ABI 1000 для создания потоков галлия и индия. Подложка InP c ориентацией (001) легирована серой до уровня $1 \cdot 10^{17} \mathrm{~cm}^{-3}$. В качестве планаризующего слоя использован $\mathrm{In}_{0.53} \mathrm{Ga}_{0.47} \mathrm{As}$ толщиной $500 \mathrm{~nm}$ и уровнем легирования $n=5 \cdot 10^{16} \mathrm{~cm}^{-3}$. Активная область включает 50 квантовых каскадов, сформированных на основе гетеропары $\mathrm{In}_{0.53} \mathrm{Ga}_{0.47} \mathrm{As} / \mathrm{In}_{0.52} \mathrm{Al}_{0.48} \mathrm{As}$. Конструкция с двухфононным резонансным рассеянием носителей заряда использована при построении активной области $[13,14]$. Для исследования конструкции волновода с тонкой верхней обкладкой толщина слоя InP уменьшена до $750 \mathrm{~nm}\left(n=1 \cdot 10^{17} \mathrm{~cm}^{-3}\right)$ в сравнении с конструкциями волновода с толстой верхней обкладкой [15]. Толщина фосфида индия в $750 \mathrm{~nm}$ соответствует объему белого фосфора в крекере, предварительно перегнанного из красной фазы, что позволяет исключить шаг дополнительного преобразования во время эпитаксии и, как следствие, возможной корректировки потоков индия и галлия при последующем выращивании контактных слоев InGaAs. В качестве контактных слоев использован $\mathrm{In}_{0.53} \mathrm{Ga}_{0.47} \mathrm{As}$ толщиной $100 \mathrm{~nm}$ и $20 \mathrm{~nm}$ с уровнями легирования $1 \cdot 10^{17}$ и $1 \cdot 10^{19} \mathrm{~cm}^{-3}$ соответственно.

Определение толщин каскадов гетероструктуры ККЛ произведено методом рентгеновской дифракции [16]. Измерения спектров рентгеновской дифракции проведены вблизи симметричного рефлекса (004) InP на дифрактометре PANalytical X'PertPro в параллельной геометрии пучка рентгеновского излучения. В качестве источника излучения (мощностью $6 \mathrm{~kW}$ ) использована трубка с вращающимся медным анодом $(\lambda=0.15406 \mathrm{~nm})$. Полуширина первичного пучка не превышала 12 ”, что обес-

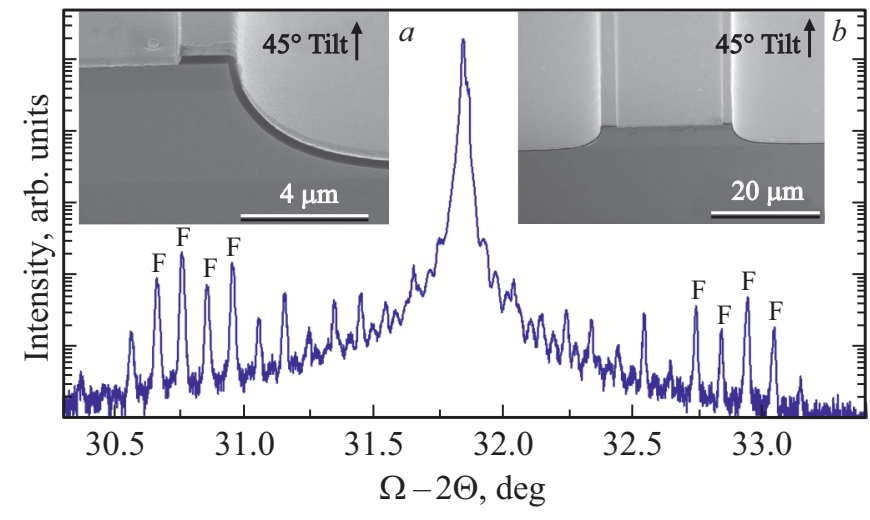

Рис. 1. Рентгенодифракционная кривая гетероструктуры ККЛ. На вставках $a$ и $b$ изображения полоскового ККЛ, полученные методом СЭМ с разным увеличением.

печивалось использованием четырехкратного $\mathrm{Ge}$ (220) прорезного кристалла-монохроматора.

Для исследования электролюминесцентных свойств ККЛ были использованы лазеры в полосковой геометрии. Формирование полоска лазера начиналось с вытравливания глубокой мезы жидкостным травлением (на основе раствора $\mathrm{HBr}: \mathrm{HNO}_{3}: \mathrm{H}_{2} \mathrm{O}$ ). В качестве маски для травления полоска использовался слой оксида кремния $\left(\mathrm{SiO}_{2}\right)$, локально вытравленный во фторсодержащей плазме под маской фоторезиста AZ 5214. После формирования профиля мезы и удаления маски на основе $\mathrm{SiO}_{2}$ производилась пассивация боковых стенок мезы путем напыления слоя нитрида кремния $\left(\mathrm{Si}_{3} \mathrm{~N}_{4}\right)$ методом плазмохимического осаждения. Толщина слоя $\mathrm{Si}_{3} \mathrm{~N}_{4}$ составила $300 \mathrm{~nm}$. Открытие окна в слое $\mathrm{Si}_{3} \mathrm{~N}_{4}$ под металлизацию проводилось путем реактивного ионного травления в смеси газов $\mathrm{CHF}_{3} / \mathrm{O}_{2}$ под маской из фоторезиста. Ширина окна составила $18 \mu \mathrm{m}$. Верхняя металлизация формировалась за счет высоковакуумного напыления (через маску фоторезиста AZ 2070) последовательных слоев титана и золота с толщинами 5 и $200 \mathrm{~nm}$ соответственно. Перед напылением нижнего металлического контакта пластина утончалась до толщины $150 \mu \mathrm{m}$. В качестве нижней металлизации использовалась последовательность слоев титан/золото с толщинами 5 и $200 \mathrm{~nm}$ соответственно. Ширина полоска вблизи поверхности гетероструктуры составила $22 \mu \mathrm{m}$, длина резонатора $1.5 \mathrm{~mm}$. Изображение скола изготовленного ККЛ, полученное методом сканирующей электронной микроскопии под углом $45^{\circ}$, представлено на вставках рис. 1. Зеркала лазеров формировались скалыванием. Отражающее и антиотражающее покрытия на сколотые грани лазера не наносились. Монтаж производился на медный теплоотвод.

Исследования смонтированных на теплоотвод экспериментальных образцов ККЛ проводились в температурном диапазоне $78-300 \mathrm{~K}$. Вольт- и ватт-амперные характеристики измерялись при пропускании импульсов тока с длительностью $100 \mathrm{~ns}$ и частотой повторения 

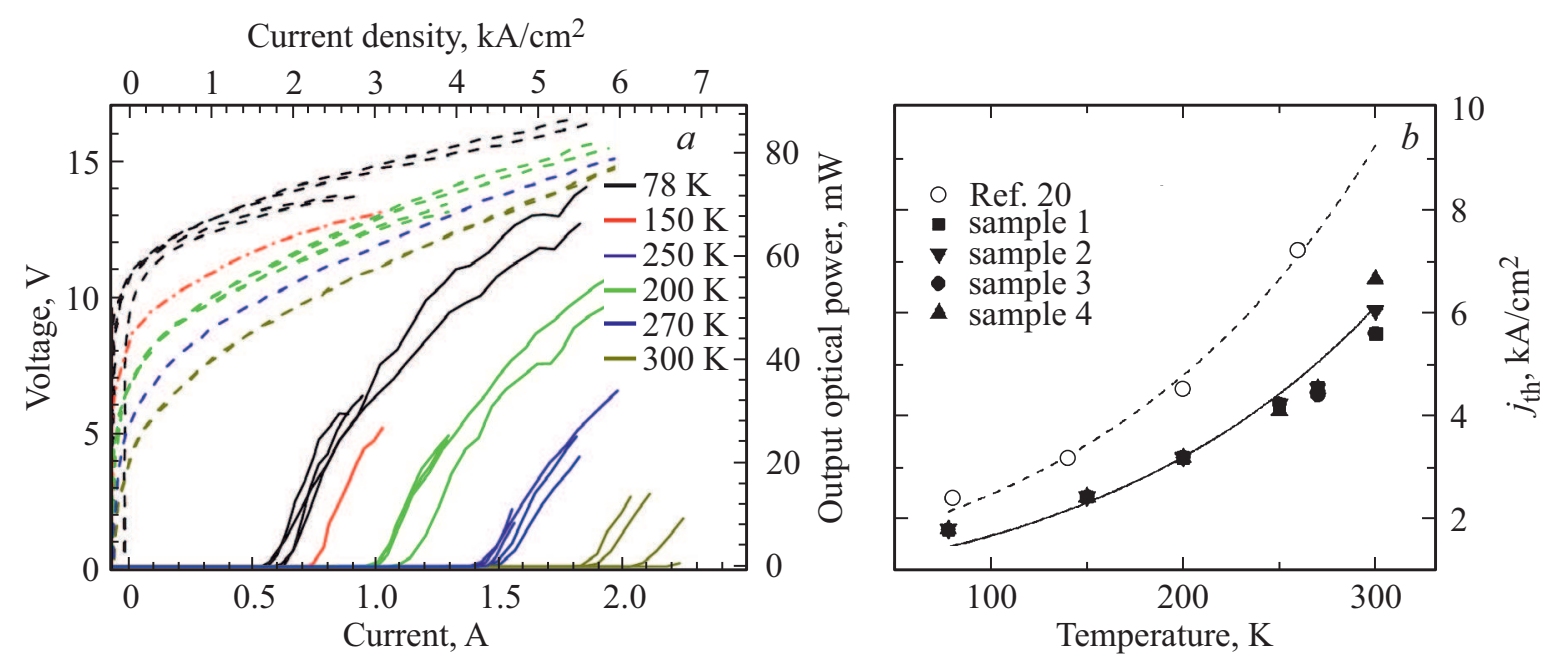

Рис. 2. $a$ - Вольт-амперные (левая ось $Y$ ) и ватт-амперные (правая ось $Y$ ) характеристики, измеренные при различных температурах; $b$ - температурные зависимости плотности порогового тока ККЛ для четырех исследованных образцов ККЛ. Сплошная кривая - результат аппроксимации экспериментальных значений. Для сравнения приведены значения (полые круги) плотности порогового тока из работы [20] и аппроксимация значений (штриховая линия).

$84 \mathrm{kHz}$. Спектры стимулированного излучения ККЛ регистрировались с помощью фурье-спектрометра и фотоприемника на основе $\mathrm{HgCdTe}$.

\section{Результаты и их обсуждение}

Рентгенодифракционная кривая исследованной гетероструктуры приведена на рис. 1. На зависимости наблюдается наличие характерного пика от подложки и слоев, формирующих верхнюю обкладку. Также присутствует последовательность пиков-сателлитов от периодической структуры каскадов, расположенных слева и справа от пика подложки. На основании положения пиковсателлитов (буквами „ „F“ на рис. 1 отмечены пикисателлиты, учтенные при расчете периода сверхрешетки) определена средняя толщина каскадов, которая составила $52 \pm 1 \mathrm{~nm}$, что соотносится с данными ростового дизайна гетероструктуры $(52.7 \mathrm{~nm})$.

Типичные вольт- и ватт-амперные характеристики, измеренные при различных температурах $(T)$, представлены на рис. 2, $a$. Изломы на ватт-амперных характеристиках связаны с конкурированием излучения нескольких мод [17]. Значение порогового напряжения $V_{\text {th }}[18,19]$ составляет $13-14 \mathrm{~V}$ при $T=78 \mathrm{~K}$ и $14-15 \mathrm{~V}$ при $300 \mathrm{~K}$ соответственно. Зависимости пороговой плотности тока $\left(j_{\text {th }}\right)$ от температуры для четырех исследованных образцов представлены на рис. 2, $b$. При $T=300 \mathrm{~K}$ минимальная величина $j_{\text {th }}$ составляет $5.8 \mathrm{kA} / \mathrm{cm}^{2}$. Таким образом, использование конструкции с тонкой верхней обкладкой позволило реализовать комнатную лазерную генерацию со стандартной схемой сплошной верхней металлизации на основе Ti/Au в сравнении с результатами для ККЛ в конструкции волновода без верхней обкладки (максимальная рабочая температура составила
$260 \mathrm{~K})[20,21]$. Экстраполяция в высокотемпературную область на основе выражения $j_{\mathrm{th}}(T)=j_{0} \exp \left(T / T_{0}\right)$ дает величину $j_{\text {th }}(T=300 \mathrm{~K}) \sim 9 \mathrm{kA} / \mathrm{cm}^{2}$ (см. результат экстраполяции на рис. 2, $b$, штриховая линия) $[20,21]$, что не позволило достичь комнатной генерации в конструкции волновода без верхней обкладки. Несмотря на тот факт, что замена золота на серебро, вносящее меньшие потери, позволила достичь комнатной лазерной генерации с значением $j_{\text {th }}(T=300 \mathrm{~K})=6 \mathrm{kA} / \mathrm{cm}^{2}$ [22], в силу низкой адгезии $\mathrm{Ag}$, его подверженности к окислению данная схема металлизации редко используется на практике. Аппроксимация на основе выражения $j_{\mathrm{th}}(T)=j_{0} \cdot \exp \left(T / T_{0}\right)[16]$ (сплошная линия рис. $\left.2, b\right)$ для исследованных образцов дает значения характеристической температуры стабильности порогового тока $T_{0}$ порядка $150 \mathrm{~K}$, плотности порогового тока при нулевой температуре $j_{0}=0.9 \mathrm{kA} / \mathrm{cm}^{2}$. Полученные значения $T_{0}$ типичны для конструкций активной области с высоким уровнем легирования инжектора $[23,24]$. На основе анализа ватт-амперных характеристик определены значения дифференциальной эффективности (Slope efficiency) для различных температур. Дифференциальная эффективность, соответствующая температуре $300 \mathrm{~K}$, составляет $0.07 \mathrm{~A} / \mathrm{W}$. Аппроксимация температурной зависимости дифференциальной эффективности на основе обратно-экспоненциальной зависимости (Slope efficiency $=$ const $\left.\cdot \exp \left(-T / T_{1}\right)\right)$ [25] позволила определить значение характеристической температуры $T_{1}$, которая составила порядка $450 \mathrm{~K}$. Типичные значения $T_{1}$ составляют порядка $300-330$ К для случая применения конструкции активной области с высоким уровнем легирования инжектора $[23,26,27]$. Таким образом, полученные в ходе исследования высокие значения $T_{1}$ характеризуют малую величину паразитной токовой утечки, обусловленную надбарьерным выбросом носи- 
телей заряда в непрерывный спектр [23]. Также проведены исследования максимально достижимой выходной оптической мощности при температуре $78 \mathrm{~K}$ (рис. 3). Лазерная генерация наблюдается при плотностях тока $j=6 j_{\text {th }}=12 \mathrm{kA} / \mathrm{cm}^{2}$, при этом выходная оптическая мощность составляет порядка $260 \mathrm{~mW}$. Стоит отметить, что в исследованных ККЛ при больших плотностях тока не наблюдается участка отрицательного дифференциального сопротивления на ВАХ, обусловленного нарушением условия резонансного туннелирования при большой напряженности электрического поля $[28,29]$. Оценочное значение выходной оптической мощности при температуре $300 \mathrm{~K}$ составляет $80-100 \mathrm{~mW}$.

На рис. 4 представлены спектры лазерной генерации, измеренные при различных температурах. С увеличе-

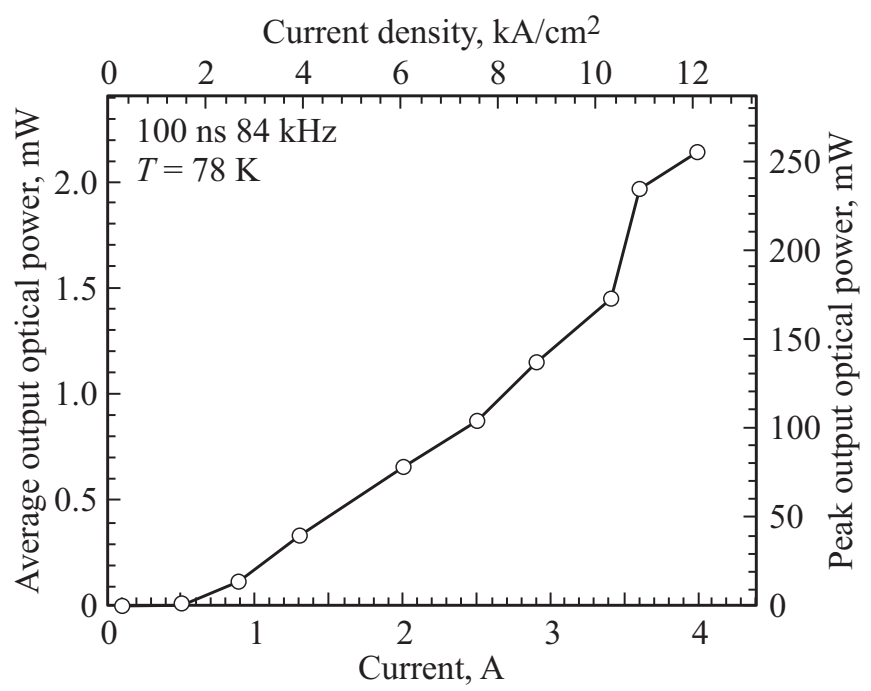

Рис. 3. Зависимость средней (левая ось $Y$ ) и пиковой (правая ось $Y$ ) выходной оптической мощности от тока накачки, измеренная при температуре $78 \mathrm{~K}$.

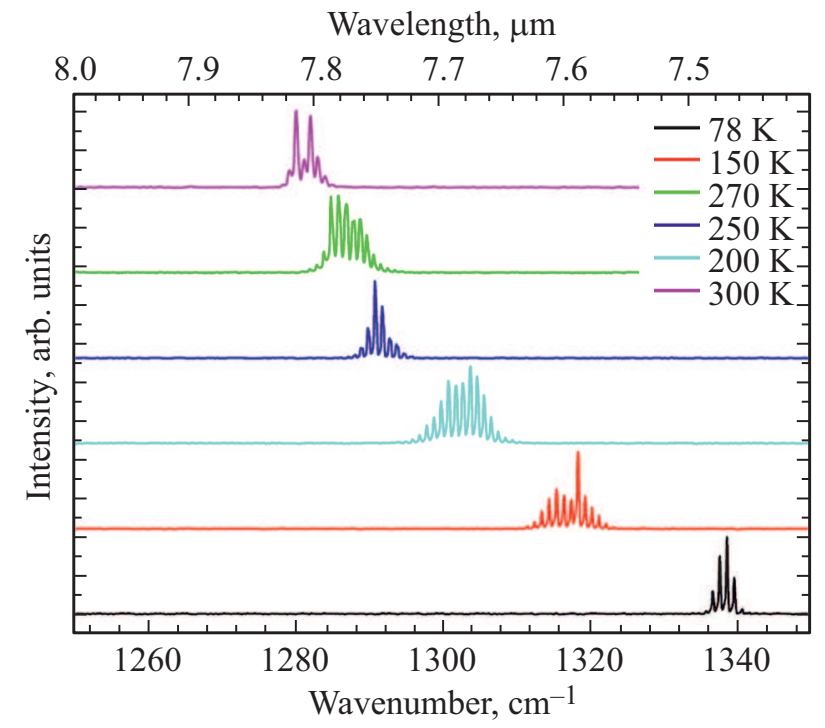

Pис. 4. Спектры стимулированного излучения образцов ККЛ, измеренные при различных температурах. нием температуры наблюдается сдвиг максимума интенсивности спектра лазерной генерации в длинноволновую область, обусловленный уменьшением разрыва зоны проводимости на гетерогранице с ростом температуры [29]. Максимум интенсивности, измеренный при $T=300 \mathrm{~K}$, соответствует длине волны $7.8 \mu \mathrm{m}$.

Произведена оценка максимальной температуры, соответствующей непрерывному режиму лазерной генерации на основе выражения $T_{\text {sub,max }}=$ $=T_{0}\left(\ln \left(T_{0} /\left(R_{\mathrm{th}} J_{0} V A\right)\right)-1\right)$, где $R_{\mathrm{th}}-$ обратная теплопроводность, $V$ - смещение на образце, $A-$ площадь лазера $[30,31]$. Типичное значение $R_{\text {th }}$ составляет $6.6-8.0 \mathrm{~K} / \mathrm{W}[32]$. Величина $j_{0}=J_{0} A=0.12 \mathrm{~A}$. Как следствие, $\quad T_{\text {sub, } \max }=170\left(\ln \left(170 /\left(R_{\mathrm{th}} \cdot 0.12 \cdot 15\right)\right)-1\right)$ может находиться в пределе $250-280 \mathrm{~K}$ в зависимости от величины $R_{\mathrm{th}}$. Величина $j_{0}$ составляет $0.3 \mathrm{kA} / \mathrm{cm}^{2}$, что свидетельствует о возможности реализации лазерной генерации в непрерывном режиме [33].

\section{Заключение}

В ходе работы продемонстрирована лазерная генерация полосковых ККЛ в конструкции волновода с тонкой верхней обкладкой InP. Все слои гетероструктуры выращены методом МПЭ на подложках InP. Лазерная генерация при температуре $300 \mathrm{~K}$ соответствует длине волны излучения $7.8 \mu \mathrm{m}$. Пороговая плотность тока $\sim 6 \mathrm{kA} / \mathrm{cm}^{2}$. Значения характеристических температур $T_{0}$ и $T_{1}$ составляют порядка 150 и $450 \mathrm{~K}$ соответственно. Максимальная выходная оптическая мощность оценена на уровне $80-100 \mathrm{~mW}$ при $T=300 \mathrm{~K}$. В сравнении с конфигурацией волновода без верхней обкладки [20,21] использование тонкой верхней обкладки позволило достичь лазерной генерации при $T=300 \mathrm{~K}$. Конфигурация с сплошной верхней металлизацией является предельным случаем с точки зрения вносимых потерь в сравнении с латеральной схемой накачки (воздушная обкладка) [13]. Тот факт, что ККЛ работает при комнатной температуре со сплошной верхней металлизацией, позволяет сделать вывод о том, что на основе данной конструкции волновода возможно формирование эффективных лазеров с распределенной обратной связью, построенных как на основе поверхностной металлической решетки [34], так и на основе латеральной схемы накачки решетки в сильнолегированном слое InGaAs [35].

\section{Финансирование работы}

Работа выполнена при частичной финансовой поддержке РФФИ (проект № 16-29-03289).

\section{Конфликт интересов}

Авторы заявляют, что у них нет конфликта интересов. 


\section{Список литературы}

[1] Yu J.S., Slivken S., Razeghi M. // Semicond. Sci. Technol. 2010. V. 25. P. 125015. doi 10.1088/0268-1242/25/12/125015

[2] Hildenbrand J., Herbst J., Wóllenstein J., Lambrecht A. // Proc. SPIE. 2009. V. 7222. P. 72220B. doi 10.1117/12.808976

[3] Michel A.P.M., Kapit J., Witinski M.F., Blanchard R. // Appl. Opt. 2017. V. 56. P. E23. doi 10.1364/ao.56.000e23

[4] Gmachl C., Straub A., Colombelli R., Capasso F., Sivco D.L., Sergent A.M., Cho A.Y. // IEEE J. Quant. Electron. 2002. V. 38. P. 569. doi 10.1109/jqe.2002.1005407

[5] Lee B.G., Belkin M.A., Audet R., MacArthur J., Diehl L., Pflügl C., Capasso F., Oakley D.C., Chapman D., Napoleone A., Bour D., Corzine S., Höfler G., Faist J. // Appl. Phys. Lett. 2007. V. 91. P. 231101. doi 10.1063/1.2816909

[6] Moreau V., Colombelli R., Perahia R., Painter O., Wilson L.R., Krysa A.B. // Opt. Express. 2008. V. 16. P. 6387. doi 10.1364/oe.16.006387

[7] Spott A., Peters J., Davenport M.L., Stanton E.J., Merritt C.D., Bewley W.W., Vurgaftman I., Kim C.S., Meyer J.R., Kirch J., Mawst L.J., Botez D., Bowers J.E. // Optica. 2016. V. 3. P. 545. doi 10.1364/optica.3.000545

[8] Jung S., Kirch J., Kim J.H., Mawst L.J., Botez D., Belkin M.A. // Appl. Phys. Lett. 2017. V. 111. P. 211102. doi $10.1063 / 1.5002157$

[9] Spott A., Stanton E.J., Volet N., Peters J.D., Meyer J.R., Bowers J.E. // IEEE J. Sel. Top. Quant. Electron. 2017. V. 23. P. 1. doi 10.1109/jstqe.2017.2697723

[10] Spott A., Stanton E.J., Torres A., Davenport M.L., Canedy C.L., Vurgaftman I., Kim M., Kim C.S., Merritt C.D., Bewley W.W., Meyer J.R., Bowers J.E. // Optica. 2018. V. 5. P. 996. doi 10.1364/optica.5.000996

[11] Jung S., Palaferri D., Xu J., Xie F., Okuno Y., Pinzone C., Lascola K., Belkin M.A. Monolithic integration of midinfrared quantum cascade lasers coupled with passive InGaAs waveguides // CLEO: Science and Innovations. 2018. San Jose. CA. US. 13-18 May 2018. ISBN: 978-1-943580-42-2. doi 10.1364/cleo_si.2018.sf2g.5

[12] Gilles C., Maisons G., Simozrag B., Carras M. // Proc. SPIE. 2015. V. 9370. P. 93702 W. doi $10.1117 / 12.2176531$

[13] Babichev A.V., Gladyshev A.G., Kurochkin A.S., Kolodeznyi E.S., Sokolovskii G.S., Bougrov V.E., Karachinsky L.Ya., Novikov I.I., Bousseksou A., Egorov A.Yu. // Semiconductors. 2018. V. 52. P. 1082. doi 10.1134/S1063782618080031

[14] Babichev A.V., Gladyshev A.G., Filimonov A.V., Nevedomskii V.N., Kurochkin A.S., Kolodeznyi E.S., Sokolovskii G.S., Bugrov V.E., Karachinsky L.Ya., Novikov I.I., Bousseksou A., Egorov A.Yu. // Tech. Phys. Lett. 2017. V. 43. P. 666. doi $10.1134 / \mathrm{s} 1063785017070173$

[15] Figueiredo P., Suttinger M., Go R., Todi A., Shu H., Tsvid E., Patel C.K.N., Lyakh A. // IEEE Photonics Technol. Lett. 2017. V. 29. P. 1328. doi 10.1109/1pt.2017.2722039

[16] Egorov A.Y., Babichev A.V., Karachinsky L.Y., Novikov I.I., Nikitina E.V., Tchernycheva M., Sofronov A.N., Firsov D.A., Vorobjev L.E., Pikhtin N.A., Tarasov I.S. // Semiconductors. 2015. V. 49. P. 1527. doi 10.1134/s106378261511007x

[17] Troccoli M. // Opt. Eng. 2010. V. 49. P. 111106. doi $10.1117 / 1.3498778$

[18] Razeghi M. // IEEE J. Sel. Top. Quant. Electron. 2009. V. 15. P. 941. doi 10.1109/jstqe.2008.2006764
[19] Babichev A.V., Bousseksou A., Pikhtin N.A., Tarasov I.S., Nikitina E.V., Sofronov A.N., Firsov D.A., Vorobjev L.E., Novikov I.I., Karachinsky L.Ya., Egorov A.Y. // Semiconductors. 2016. V. 50. P. 1299. doi 10.1134/s1063782616100067

[20] Bousseksou A., Colombelli R., Babuty A., De Wilde Y., Chassagneux Y., Sirtori C., Patriarche G., Beaudoin G., Sagnes I. // Opt. Express. 2009. V. 17. P. 9391. doi 10.1364/oe.17.009391

[21] Bousseksou A., Chassagneux Y., Colombelli R., Babuty A., De Wilde Y., Sirtori C., Patriarche G., Beaudoin G., Sagnes I. // LEOS 2008 - 21st Annual Meeting of the IEEE Lasers and Electro-Optics Society, Nov. 2008. Acapulco, Mexico. P. 804. doi 10.1109/LEOS.2008.4688865

[22] Bahriz M., Moreau V., Palomo J., Colombelli R., Austin D.A., Cockburn J.W., Wilson L.R., Krysa A.B., Roberts J.S. // Appl. Phys. Lett. 2006. V. 88. P. 181103. doi 10.1063/1.2198016

[23] Botez D., Kirch J.D., Boyle C., Oresick K.M., Sigler C., Kim H., Knipfer B.B., Ryu J.H., Lindberg D., Earles T., Mawst L.J., Flores Y.V. // Opt. Mater. Express. 2018. V. 8. P. 1378. doi 10.1364/ome.8.001378

[24] Yu J.S., Slivken S., Razeghi M. // Semicond. Sci. Technol. 2010. V. 25. P. 125015. doi 10.1088/0268-1242/25/12/125015

[25] Dudelev V.V., Losev S.N., Mylnikov V.Yu., Babichev A.V., Kognovitskay E.A., Slipchenko S.O., Lyutetsky A.V., Pikhtin N.A., Gladyshev A.G., Karachinsky L.Ya., Novikov I.I., Egorov A.Yu., Kuchinsky V.I., Sokolovskii G.S. // Phys. Solid State. 2018. V. 60. P. 2291. doi 10.1134/S1063783418110057

[26] Liu P.Q., Hoffman A.J., Escarra M.D., Franz K.J., Khurgin J.B., Dikmelik Y., Wang X., Fan J.-Y., Gmachl C.F. // Nat. Photonics. 2010. V. 4. P. 95. doi 10.1038/nphoton.2009.262

[27] Maulini R., Lyakh A., Tsekoun A., Patel C.K.N. // Opt. Express. 2011. V. 19. P. 17203. doi 10.1364/oe.19.017203

[28] Teissier R., Barate D., Vicet A., Yarekha D.A., Alibert C., Baranov A.N., Marcadet X., Garcia M., Sirtori C. // Electron. Lett. 2003. V. 39. P. 1252. doi 10.1049/el:20030780

[29] Bai Y., Slivken S., Kuboya S., Darvish S.R., Razeghi M. // Nat. Photonics 2010. V. 4. P. 99. doi 10.1038/nphoton.2009.263

[30] Faist J., Capasso F., Sirtori C., Sivco D.L., Hutchinson A.L., Cho A.Y. // Appl. Phys. Lett. 1995. V. 67. P. 3057. doi 10.1063/1.114863

[31] Faist J., Tredicucci A., Capasso F., Sirtori C., Sivco D.L., Baillargeon J.N., Hutchinson A.L., Cho A.Y. // IEEE J. Quant. Electron. 1998. V. 34. P. 336. doi 10.1109/3.658728

[32] Colombelli R., Tredicucci A., Gmachl C., Capasso F., Sivco D.L., Sergent A.M., Hutchinson A.L., Cho A.Y. // Electron. Lett. 2001. V. 37. P. 1023. doi 10.1049/el:20010694

[33] Faist J., Capasso F., Sirtori C., Sivco D.L., Hutchinson A.L., Chu S.-N.G., Cho A.Y. // Superlattices Microstruct. 1996. V. 19. P. 337. doi 10.1006/spmi. 1996.0037

[34] Carras M., Garcia M., Marcadet X., Parillaud O., De Rossi A., Bansropun S. // Appl. Phys. Lett. 2008. V. 93. P. 011109. doi 10.1063/1.2950086

[35] Hofstetter D., Faist J., Beck M., Müller A., Oesterle U. // Appl. Phys. Lett. 1999. V. 75. P. 665. doi 10.1063/1.124475 